\title{
Ein Fall von doppelseitigem Hydrops genu intermittens.
}

Von Dr. te Kanp in Bad Salzschlirf.

Die unter dem Namen Hydrops articulorum intermittens bekannte Gelenkaffektion gehört zu den sehr selten vorkommenden; sie befällt am häufigsten das Kniegelenk und tritt in ziemlich regelmäBigen Intervallen auf. Folgenden von mir beobachteten Fall von abwechselnd in beiden Kniegelenken auftretendem Hydrops intermittens möchte ich seines typischen Verhaltens und der Seltenheit der Affektion wegen kurz mitteilen.

Eine ältere Dame konsultierte mich wegen rheumatisch-gichtischer Beschwerden der Knie- und Hüftgelenke; sie gibt an, seit Jahren an einer wechselweise auftretenden An- und Abschwellung der Kniegelenke zu leiden; die Intervalle hätten früher neun Tage betragen, jetzt betrügen sie nur mehr vier Tage.

Objektiver Befund bei der ersten Untersuchung: Der Umfang beider Kniegelenke in Streckstellung über der Mitte der Patella gemessen, ist beiderseits gleich groß und beträgt $45 \mathrm{~cm}$. Die Exkursionsfähigkeit beider Kniegelenke ist normal. Im rechten Kniegelenk fühlt man bei Bewegung desselben etwas Crepitation.

Einige Tage später stellte sich die Patientin wieder vor. Befund: Der Umfang des rechten Kniegelenks, in Streckstellung gemessen, beträgt etwa $49 \mathrm{~cm}$; Hydrops genu; Bewegungsfähigkeit des Gelenks fast aufgehoben. Der Umfang des linken Kniegelenks ist wie bei der ersten Untersuchung $45 \mathrm{~cm}$.

Sechs Tage später stellt sich Patientin zum dritten Male vor. Befund: Rechtes Knie $46 \mathrm{~cm}$ Umfang; Hydrops nicht mehr nachweisbar; Gelenk ziemlich gut beweglich. Umfang des linken Kniegelenks in Streckstellung $47 \mathrm{~cm}$; heute ist der erste Erkrankungstag für das linke Kniegelenk, bis zum nächsten Tage wird die Schwellung infolge Zunahme des Hydrops noch vermehrt sein, wohingegen (nach Angabe der Patientin) das zuerst affizierte rechte Kniegelenk bis dahin wieder ganz normal ist; im übrigen ist das rechte Knie immer etwas stärker geschwollen.

Also alle 6-7 Tage kommt es zu einem ziemlich plötzlich innerhalb $24-36$ Stunden auftretenden Hydrops genu eines Beines; der Hydrops bleibt drei Tage lang auf seinem Höhepunkte stehen, vom dritten bis sechsten Tage nimmt die Gelenkschwellung wieder $\mathrm{ab}$, und am sechsten oder siebenten Tage tritt dieselbe Affektion im andern Knie auf.

Diese Affektion, die ohne Fieber und mit nur geringen Schmerzen (nicht schmerzlos in vorliegendem Falle!) einhergeht, hat mit Rheumatismus und Gicht nichts zu tun. Sie ist in ihren Ursachen noch unbekannt und wird meist als eine vasomotorischtrophische Gelenkneurose aufgefaßt, allerdings wohl mit wenig Recht. Das Leiden dauert jahrzehntelang, vielleicht lebenslänglich, kann lange Zeit aussetzen und dadurch eine Heilung vortäuschen. Die Therapie ist machtlos, wenigstens erwiesen sich Trink- und Badekuren, sowie Salicyl, Chinin, Arsenik, auch hydropathische Behandlung als wirkungslos. 